\title{
Cost-Effectiveness Analysis of First-Line Cetuximab Plus Leucovorin, Fluorouracil, and Oxaliplatin (FOLFOX-4) versus FOLFOX-4 in Patients with RAS Wild-Type Metastatic Colorectal Cancer
}

This article was published in the following Dove Press journal:

Cancer Management and Research

\author{
Liangliang $\mathrm{Bai}^{1,2}$ \\ Pengfei Zhang ${ }^{1,2}$ \\ Kexun Zhou ${ }^{1,2}$ \\ Weiting Liao ${ }^{1,2}$ \\ Qiu $\mathrm{Li}^{1,2}$
}

'Department of Medical Oncology, Cancer Center, West China Hospital, Sichuan University, Chengdu, Sichuan 6I004I, People's Republic of China; ${ }^{2}$ West China Biomedical Big Data Center, Sichuan University, Chengdu, Sichuan 61004I, People's Republic of China
Correspondence: Qiu Li

Department of Medical Oncology, Cancer Center, West China Hospital, Sichuan University, No.37, GuoXue Xiang, Chengdu, Sichuan 61004I, People's Republic of China

Tel +862885423262

Fax +86 2885423609

Email liqiu@scu.edu.cn
Purpose: Compared with fluorouracil, leucovorin, and oxaliplatin (FOLFOX-4) alone, cetuximab plus FOLFOX-4 has shown superior performance in terms of efficacy and tolerability in patients with RAS wide-type (wt) metastatic colorectal cancer (mCRC) in the TAILOR trial (Trial No.: EMR62202-057; ClinicalTrials.gov identifier: NCT01228734). Thus, we aimed to explore the cost-effectiveness of these two first-line regimens in patients with RAS wt mCRC from the Chinese societal perspective.

Methods: For the sake of executing the analysis, we used a Markov model containing three health states (progression-free survival (PFS), progressive disease (PD), and death) to simulate the process of RAS wt mCRC. The data regarding efficacy and safety were derived from the TAILOR trial. Transition probabilities were converted from the PFS and overall survival (OS) of both groups. Utility scores of the health states were obtained from previously published studies. Costs were computed from the perspective of Chinese society. The primary health outcome was the incremental cost-effectiveness ratio (ICER). Sensitivity analysis was utilized to investigate the effect of uncertainties on the Markov model.

Results: Treatment with cetuximab plus FOLFOX-4 was estimated to provide an increase in quality adjusted-life years (QALYs) of 0.15 QALYs at an increased cost of $\$ 19,079$ compared with FOLFOX-4 alone, resulting in an ICER of \$127,193/QALY, which exceeded the threshold of willingness-to-pay (WTP) of \$27,934/QALY in China. Sensitivity analysis showed that the cost of PFS in the cetuximab plus FOLFOX-4 arm was the most influential factor in the Markov model.

Conclusion: The combination of cetuximab and FOLFOX-4 is not a cost-effective strategy compared with FOLFOX-4 alone for the first-line treatment of patients with RAS wt mCRC from the perspective of Chinese society.

Keywords: cost-effectiveness, metastatic colorectal cancer, cetuximab, FOLFOX-4

\section{Introduction}

Colorectal cancer (CRC) is a common malignant tumor of the digestive tract. According to the GLOBOCAN estimates for 2018, CRC ranked third in terms of incidence and was the second leading cause of cancer-related mortality. ${ }^{1}$ In the past, therapies combining a fluorouracil, leucovorin and irinotecan (FOLFIRI) regimen with an anti-vascular endothelial growth factor (VEGF) monoclonal antibody or antiepidermal growth factorg receptor (EGFR) monoclonal antibody improved survival 
and were standard first-line treatments. ${ }^{2-4}$ However, it was still controversial to use anti-EGFR monoclonal antibody cetuximab plus oxaliplatin-based chemotherapy for the firstline treatment of patients with RAS wide-type (wt) metastatic colorectal cancer (mCRC). ${ }^{5-8}$ With the report of the results of the TAILOR trial (Trial No.: EMR62202-057; ClinicalTrials. gov identifier: NCT01228734), this controversy was resolved. ${ }^{9}$

The TAILOR trial was the first prospective, open-label, randomized, multicenter, phase III study to confirm the efficacy and safety of adding cetuximab to first-line fluorouracil, leucovorin and oxaliplatin (FOLFOX-4) in patients with RAS wt mCRC. The results clearly demonstrated that adding cetuximab to FOLFOX-4 significantly improved the median progression-free survival ( $\mathrm{PFS})(\mathrm{P}=0.004$, median PFS, 9.2 months vs 7.4 months), median overall survival (OS) ( $\mathrm{P}=0.02$, median OS, 20.7 months vs 17.8 months) and overall response rate $(\mathrm{P}<0.001$, ORR, $61.1 \%$ vs $39.5 \%)$ compared with FOLFOX-4 alone. Meanwhile, the treatment was well tolerated, and the safety profile of cetuximab plus FOLFOX-4 was in accordance with expectations. ${ }^{9}$ In addition, unlike in the CALGB/SWOG 80405 trial, it seemed that patients with right-sided, BRAF wt mCRC could benefit from the addition of cetuximab to first-line FOLFOX-4 in the TAILOR trial. ${ }^{9,10}$

Although therapy with cetuximab plus FOLFOX-4 shows certain advantages, health care costs increased significantly in the process of treatment. In China with limited health resources and a large population, health care payers and clinicians also need dependable evidence as a framework for determining the value of different therapeutic regimens in oncology. Therefore, we used a Markov model to explore the cost-effectiveness of cetuximab plus FOLFOX-4 compared with FOLFOX-4 alone for patients with RAS wt mCRC from the perspective of Chinese society.

\section{Materials and Methods}

\section{Patients and Regimens}

The basic clinical data were derived from the literature of TAILOR trial. ${ }^{9}$ FOLFOX-4 consisted of intravenous oxaliplatin $\left(85 \mathrm{mg} / \mathrm{m}^{2}\right)$ on day 1 , leucovorin $\left(200 \mathrm{mg} / \mathrm{m}^{2}\right)$ on days $1-2$ and 5-fluorouracil (bolus $400 \mathrm{mg} / \mathrm{m}^{2}$ and then a 22 hours continuous infusion of $600 \mathrm{mg} / \mathrm{m}^{2}$ ) on days $1-2$ of each 2-week treatment cycle. For patients receiving cetuximab plus FOLFOX-4, cetuximab was administered at $400 \mathrm{mg} / \mathrm{m}^{2}$ on day 1 and then at $250 \mathrm{mg} / \mathrm{m}^{2} /$ week. According to the results of treatment exposure of the PFS state, doses of cetuximab, oxaliplatin and 5-fluorouracil were adjusted based on the observed changes in adverse events (AEs) during treatment. To assess the tumor response, computed tomography (CT) or magnetic resonance imaging (MRI) was conducted every 8 weeks, and follow-up was carried out every 3 months until the patient died. The baseline characteristics of the patients with RAS wt mCRC were reasonably balanced between the two arms.

\section{Model Structure}

A Markov cohort simulation model was conducted by TreeAge Pro 2011 (TreeAge Software, Inc., Williamstown, MA, USA) to simulate the evaluation of patients health with RAS wt mCRC and to explore the cost-effectiveness of these two regimens based on the TAILOR trial. Markov models are commonly used in cost-effectiveness analysis evaluating cancer therapies. The Markov model simulates the progression of the patient health through three health states: PFS, progressive disease (PD), and death (Figure 1). All patients first enter the model in the PFS state. Each month, they can transition to another health state (Figure 1). The monthly transition probabilities of the three health states were calculated as follows: $P(1$ month $)=1-(0.5)^{(1 / \text { median time to event })}$, which was derived from the equations: $\mathrm{P}=1-\mathrm{e}-\mathrm{R}$ and $\mathrm{R}=-\ln [0.5] /$ (time to event/number of treatment cycles). And monthly transition probabilities were based on the TAILOR trial. ${ }^{11,12}$

\section{Cost Estimates}

The total costs were computed from the perspective of Chinese society, and they consisted of direct medical costs and social costs. The direct medical costs include the drugs, tests, treatments for grade $\geq 3 \mathrm{AEs}$, and hospitalization, while the social costs included travel fees and time costs. The costs

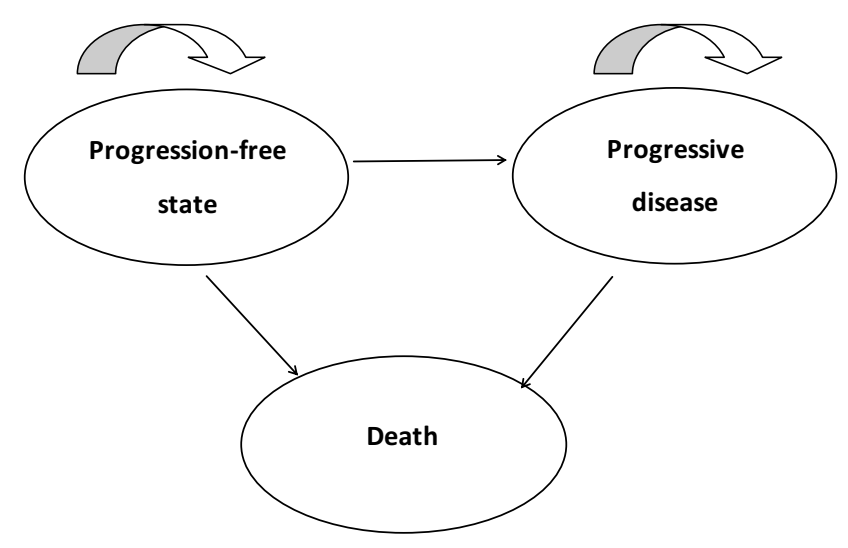

Figure I Markov model for RAS wide-type metastatic colorectal cancer. Notes: A Markov model containing three health states (progression-free state, progressive disease, and death) was conducted. 
of drugs, tests and hospitalization were in accordance with the 2018 fee standards of West China Hospital, Sichuan University. For the costs of treatments for grade $\geq 3$ AEs, we referred to the incidence of grade $\geq 3$ AEs in the population with RAS wt mCRC to calculate them (Table 1). Travel fees were estimated to be $\$ 10.20$ per patient each trip to the hospital according to the taxi fare per kilometer in Sichuan, China, 2018. ${ }^{13}$ And time costs were reckoned at $\$ 35.73$ per day on the basis of the average monthly salary in China, $2018 .^{13}$ All costs were converted into US dollars at an exchange rate of $\$ 1=$ RMB 6.9425 (November 2018).

\section{Cost-Effectiveness Estimates}

The incremental cost-effectiveness ratio (ICER) was the primary health outcome, which was associated with costs and the quality-adjusted life years (QALYs) of both groups. Utility scores of the health states in the Markov model were obtained from previously published studies, with 0.85 for the PFS state in groups, 0.68 for the PD state and 0 for death state. ${ }^{14-16}$

\section{Sensitivity Analysis}

One-way sensitivity analysis was carried out to observe the effect of different parameters on the Markov model, the range of which was $\pm 30 \%$ of the baseline values, and the results of our analysis were displayed in the form of a tornado diagram. Probabilistic sensitivity analysis using a Monte Carlo simulation of 1000 patients was performed to

Table I Clinical Efficacy and Grade 3-4 AEs

\begin{tabular}{|l|l|l|}
\hline Variables & $\begin{array}{l}\text { Cetuximab } \\
\text { +FOLFOX-4 }\end{array}$ & FOLFOX-4 \\
\hline \multicolumn{2}{|l|}{ Clinical efficacy, months (95\% Cl) } \\
\hline Median PFS (m) & $9.2(7.7-9.4)$ & $7.4(5.6-7.9)$ \\
Median OS (m) & $20.7(15.9-22.1)$ & $17.8(14.9-19.6)$ \\
\hline Grades 3-4 AEs (\%) & \multicolumn{2}{|l}{} \\
\hline Neutropenia & 61.9 & 43.2 \\
Leukopenia & 26.8 & 21.1 \\
Fatigue & 12.9 & 9.5 \\
Hypokalemia & 10.3 & 4.0 \\
Thrombocytopenia & 10.3 & 6.5 \\
Hypomagnesemia & 8.2 & 1.0 \\
Dermatitis acneiform & 7.2 & 0 \\
Stomatitis & 6.2 & 0.5 \\
Diarrhea & 5.7 & 2.0 \\
Bone marrow failure & 4.6 & 6.0 \\
Any skin reactions & 25.8 & 0 \\
\hline
\end{tabular}

Abbreviations: AEs, adverse events; FOLFOX-4, leucovorin, fluorouracil, and oxaliplatin; OS, overall survival; PFS, progression-free survival. determine the optimal strategy under the premise of varying willingness-to-pay (WTP) thresholds, and the results of our analysis were presented as cost-effectiveness acceptability curves. According to the guidelines of World Health Organization (WHO) for cost-effective analyses, WTP was set to \$27,934/QALY, which was $3 \times$ the per capita gross domestic product (GDP) of China. ${ }^{17}$

\section{Results}

\section{Basic Outcomes}

The efficacy and grade $\geq 3$ AEs are displayed in Table 1 . According to aforementioned equation, the monthly transition probability of the group receiving cetuximab plus FOLFOX-4 from the PFS state to PD state ( $\left.\mathrm{P}_{\mathrm{PFS}-\mathrm{PD} 1}\right)$ was $0.073,0.033$ from the PFS state to death $\left(\mathrm{P}_{\mathrm{PFS}-\mathrm{DEATH}}\right)$, and 0.058 from the $\mathrm{PD}$ state to death $\left(\mathrm{P}_{\mathrm{PD}-\mathrm{DEATH}}\right)$. The transition probability of the group receiving FOLFOX-4 alone from the PFS state to the PD state $\left(\mathrm{P}_{\mathrm{PFS}-\mathrm{PD} 2}\right)$ was $0.089,0.038$ from the PFS state to death $\left(\mathrm{P}_{\mathrm{PFS}-\mathrm{DEATH}}\right)$, and 0.064 from the $\mathrm{PD}$ state to death $\left(\mathrm{P}_{\mathrm{PD}-\mathrm{DEATH}}\right)$. When the Markov process ended, the combination of cetuximab and FOLFOX-4 provided 1.25 QALYs compared with 1.10 QALYs for FOLFOX-4 alone.

\section{Cost Outcomes}

The estimated monthly costs of drugs, tests, treatments for grade $\geq 3$ AEs, and hospitalization as well as the societal costs are given in Table 2. Briefly, in the cetuximab and FOLFOX-4 arm, the costs for the PFS state and PD state were $\$ 30,825$ and $\$ 9,175$, respectively. In the FOLFOX-4 arm, the costs of the PFS state and PD state were $\$ 6,372$ and $\$ 14,549$, respectively. Finally, the total cost was $\$ 40,000$ for the cetuximab and FOLFOX-4 arm, which was remarkably higher than the $\$ 20,921$ for the FOLFOX-4 arm (Table 3), and the introduction of cetuximab substantially increased the total cost by $\$ 19,079$.

\section{Cost-Effectiveness Analysis}

According to the results of cost-effectiveness analysis presented in Table 3, the combination of cetuximab and FOLFOX-4 was more expensive, with a cost of $\$ 32,000$ per QALY compared with $\$ 19,019$ per QALY for the FOLFOX-4 alone. In general, cetuximab plus FOLFOX-4 provided an increase of 0.15 QALYs at an incremental cost of $\$ 19,079$ compared with FOLFOX-4 alone, resulting in an ICER of $\$ 127,193 /$ QALY, which exceeded the WTP threshold of $\$ 27,934 /$ QALY in China. 
Table 2 Cost (\$) and Utility Scores of Cetuximab Plus FOLFOX4 and FOLFOX-4

\begin{tabular}{|c|c|c|}
\hline Variables & $\begin{array}{l}\text { Cetuximab } \\
\text { +FOLFOX-4 }\end{array}$ & FOLFOX-4 \\
\hline \multicolumn{3}{|l|}{ Cost for PFS state ( $\$ /$ month) } \\
\hline Chemotherapy drugs & 263 & 276 \\
\hline Cetuximab & 2,517 & 0 \\
\hline Test & 238 & 238 \\
\hline Grade $\geq 3$ AEs & 59 & 40 \\
\hline Inpatient fees & 123 & 92 \\
\hline Time & 41 & 20 \\
\hline Travel & 286 & 214 \\
\hline Total cost for PFS state & 3,527 & 880 \\
\hline \multicolumn{3}{|l|}{ Cost for PD state ( $\$ /$ month) } \\
\hline Chemotherapy drugs & 567 & $\mathrm{I}, 06 \mathrm{I}$ \\
\hline Additional cost for PD state & 257 & 344 \\
\hline Total cost for PD state & 824 & $\mathrm{I}, 405$ \\
\hline
\end{tabular}

Abbreviations: FOLFOX-4, leucovorin, fluorouracil, and oxaliplatin; PFS, progression-free survival; $P D$, progressive disease.

Table 3 Results of Cost-Effectiveness Analysis

\begin{tabular}{|c|c|c|}
\hline Variables & $\begin{array}{l}\text { Cetuximab } \\
\text { +FOLFOX-4 }\end{array}$ & FOLFOX-4 \\
\hline \multicolumn{3}{|l|}{ Cost $(\$)$} \\
\hline PFS state & 30,825 & 6,372 \\
\hline PD state & 9,175 & 14,549 \\
\hline Total cost & 40,000 & 20,921 \\
\hline Incremental cost & 19,079 & \\
\hline \multicolumn{3}{|l|}{ Effectiveness (QALYs) } \\
\hline PFS state & 0.62 & 0.51 \\
\hline PD state & 0.63 & 0.59 \\
\hline Total effectiveness & 1.25 & 1.10 \\
\hline Incremental effectiveness & 0.15 & \\
\hline ICER (\$/QALY) & 127,193 & \\
\hline
\end{tabular}

Abbreviations: FOLFOX-4, leucovorin, fluorouracil, and oxaliplatin; PFS, progression-free survival; PD, progressive disease; QALYs, quality adjusted-life years; ICER, incremental cost-effectiveness ratio.

\section{Sensitivity Analysis}

One-way sensitivity analysis was performed to explore the effect of uncertainties in our analysis, and the results are shown in a tornado diagram (Figure 2). The effect of the cost of the PFS state in the cetuximab plus FOLFOX-4 arm was more dominant in the Markov model. When the cost of the PFS state in the cetuximab plus FOLFOX-4 arm varied from $\$ 2,469$ to $\$ 4,585$, the ICER increased from $\$ 65,411$ per QALY to $\$ 188,445$ per QALY. In the PFS state in the cetuximab plus FOLFOX-4 arm, the proportion of the cost of cetuximab was higher. When the cost of cetuximab in the cetuximab plus FOLFOX-4 arm varied from $\$ 1,762$ to $\$ 3,272$, the ICER increased from $\$ 83,029$ per QALY to $\$ 170,827$ per QALY. The costs of the PD state in the FOLFOX-4 arm and the cetuximab plus FOLFOX-4 arm, and the utility scores of PFS also had significant impacts on ICER. In addition, according to the probabilistic sensitivity analysis, the cost-effectiveness acceptability curves indicated that the FOLFOX-4 regimen had an approximately $100 \%$ probability of being cost-effective when the WTP was set to $\$ 27,934 /$ QALY, so the cetuximab plus FOLFOX-4 regimen was not a cost-effective strategy compared with the FOLFOX-4 regimen (Figure 3).

\section{Discussion}

Currently, the combination of chemotherapy with monoclonal antibodies is the standard first-line treatment for patients with RAS wt mCRC. In the TAILOR trial, adding cetuximab to first-line FOLFOX-4 in patients with RAS wt mCRC significantly improved the ORR, PFS and OS. However, the addition of cetuximab resulted in a substantial increase in the cost of treatment. Thus, it was not clear whether the combination of cetuximab and FOLFOX-4 is a cost-effective strategy for patients with RAS wt mCRC from the perspective of Chinese society.

Our analysis based on the TAILOR trial is the first study to explore the efficacy and cost of adding cetuximab to first-line FOLFOX-4 in patients with RAS wt mCRC, who are different from the patients with KRAS wt mCRC in prior trials. ${ }^{4,6,18}$ According to our analysis, cetuximab plus FOLFOX-4 provided an incremental 0.15 QALYs at an increase of $\$ 19,079$ compared with the FOLFOX-4 group, resulting in an ICER of $\$ 127,193 /$ QALY, which exceeded the WTP threshold of $\$ 27,934 /$ QALY in China. Thus, although the results from the TAILOR phase III trial suggest that the addition of cetuximab to FOLFOX-4 showed excellent clinical benefits, it may not be a cost-effective strategy for patients with RAS wt mCRC from the Chinese societal perspective.

Regarding the cost-effectiveness of cetuximab plus FOLFOX-4, there seemed to be a consistent answer in several studies worldwide. A systematic review noted that the ICER for RAS WT mCRC patients treated with cetuximab plus FOLFOX-4 against FOLFOX-4 was $£ 104,205 /$ QALY, which was likely to represent poor value for the money at a WTP of $£ 20,000$ per QALY when judged by the costeffectiveness criteria used in the UK, ${ }^{19}$ but this result was obtained merely by subgroup analysis. In Iran, a systematic search of the literature showed that the addition of cetuximab 


\section{Tornado Analysis (ICER)}

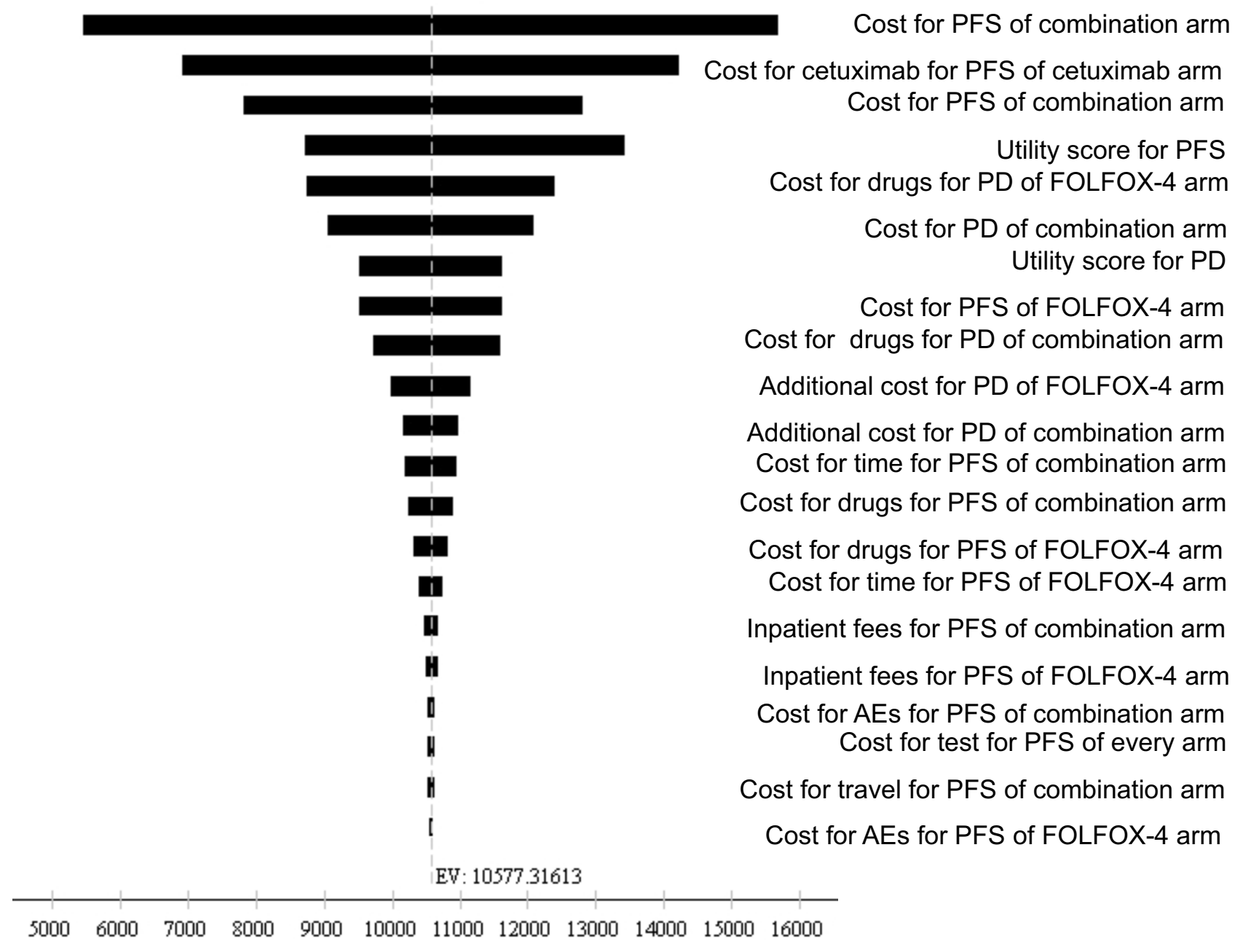

ICER for cetuximab plus FOLFOX-4 arm versus FOLFOX-4 arm (\$/QALM)

Figure 2 Tornado diagram of the one-way sensitivity analysis.

Notes: The tornado diagram showed the results of the one-way sensitivity analysis to observe the effect of different parameters on the Markov model. The parameters are arranged in descending order in terms of trend with the degree of influence on the Markov model.

Abbreviations: FOLFOX-4, leucovorin, fluorouracil, and oxaliplatin; combination arm, cetuximab plus FOLFOX-4 arm; PFS, progression-free survival; PD, progressive disease; QALM, quality adjusted-life month; ICER, incremental cost-effectiveness ratio.

to FOLFOX-4 increased OS by 0.442 years and increased cost by $\$ 202,484$, resulting in an ICER of US\$ 458,113 per life years gained (LYG), which greatly exceeded the WHO recommended threshold for the Iranian health care market, ${ }^{20}$ however a potential limitation was that the RAS gene status of the mCRC patients was unclear. Compared with previously published studies, our study addresses the defects in the design of these previous studies and similarly confirms that cetuximab plus FOLFOX-4 is not cost-effective in China. Moreover, the differences in the research perspectives and methods of these three studies may produce bias, which could further impact the cost-effectiveness of first-line cetuximab plus FOLFOX-4 vs FOLFOX-4 in patients with RAS wt mCRC in different countries.

In addition, a study from the Chinese medical insurance perspective found that the addition of cetuximab to FOLFIRI led to an increase of 0.63 QALYs at an incremental cost of $\$ 17,086$ in the treatment of patients with RAS wt mCRC. When the patient assistance program was available, the ICER decreased to \$14,049/QALY, which indicated that cetuximab is cost-effective at WTP of $\$ 22,200 /$ QALY in China in $2016^{21}$ This finding conflicts with ours; a likely explanation for this inconsistency is that we explored the cost-effectiveness of the two regimens from the perspective of Chinese society rather 


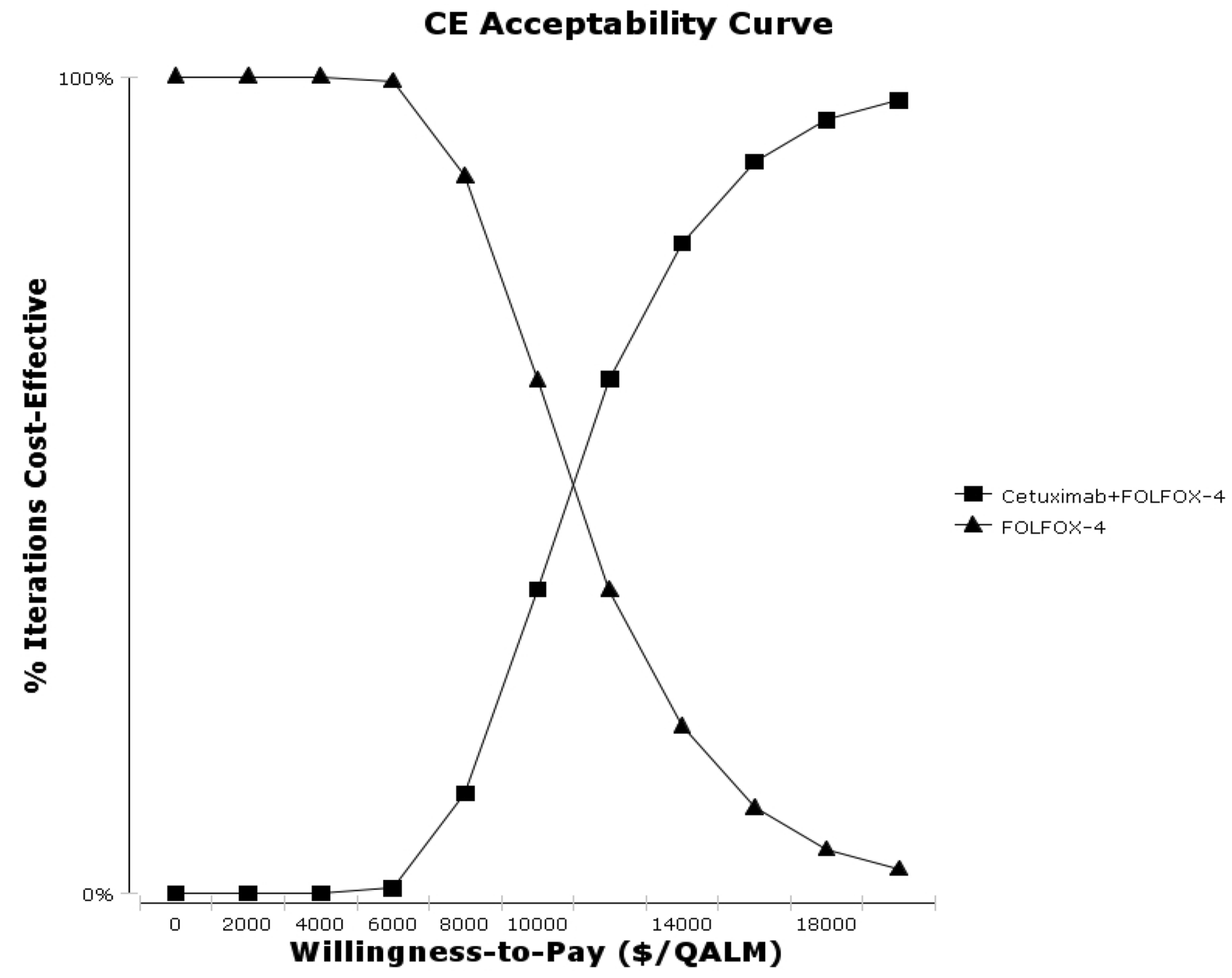

Figure 3 Cost-effectiveness acceptability curves.

Notes: The curves show the results of probabilistic sensitivity analysis to determine the optimal strategy under the premise of varying willingness-to-pay thresholds. Abbreviations: FOLFOX-4, leucovorin, fluorouracil, and oxaliplatin; CE, cost-effectiveness; QALM, quality-adjusted life month.

than the perspective of Chinese medical insurance. If WTP of China increases to $\$ 150,000$ per life year (LY), as is found in the US, adding cetuximab to FOLFOX-4 will be a costeffective regimen in patients with RAS wt mCRC in China.

In one-way sensitivity analysis, the cost of the PFS state in the cetuximab plus FOLFOX-4 arm was the most influential factor in our study, which was consistent with previously published literature. ${ }^{19,22}$ In addition, the cost of the PD state in the FOLFOX-4 arm was also influential on the Markov model, mainly because patients in the FOLFOX-4 arm were larger than that in the cetuximab plus FOLFOX-4 arm receiving later-line EGFR-targeting therapies (15\% vs 1.6\%). With negotiations in health care for anticancer drugs, the anti-EGFR monoclonal antibody cetuximab was approved for inclusion in the health care catalogue in China in October 2018, this treatment costs $\$ 186.53$ (20 mL: $100 \mathrm{mg}$ ), which is paid by health care payers. However, because of differences in the proportion of medical insurance reimbursement and the termination of an assistance program for cetuximab, the economic burden exceeding the set WTP is still large in China. Hence, the development of domestic generic drugs and the lowering of prices of molecule-targeted drugs are required to further relieve the economic burden of patients with RAS wt $\mathrm{mCRC}$ in the future, which will lead to a decline in ICER.

There were some limitations of our cost-effective analysis that deserve to be mentioned. First, some of the clinical data were retrospectively collected from TAILOR trial, which were not patient-level data in clinical practice. Therefore, the results of our study had a lower statistical power. Second, owing to a lack of relevant information about the quality of life to calculate the utility scores for the PFS and PD states in the FOLFOX-4 arm and the cetuximab plus FOLFOX-4 arm in 
TAILOR trial, we employed analogous data obtained from previously published literature to develop a Markov model to simulate the process of RAS wt mCRC. Third, compared with previously reported trials in $\mathrm{mCRC}$, the shorter OS in the TAILOR trial, which resulted from few patients receiving additional therapy after progression, reduces the incremental QALY, which directly led to an increase in the ICER.

\section{Conclusion}

Our results revealed that the combination of cetuximab and FOLFOX-4 is not a cost-effective strategy compared with FOLFOX-4 alone for patients with RAS wt mCRC when the WTP is set to $\$ 27,934 /$ QALY in China. We believe that with the development of clinical trials, increasing numbers of high-efficacy and low-price regiments will be applied to clinical treatment.

\section{Acknowledgments}

The authors gratefully acknowledge help from David Hutton, the professor of the Department of Health Management and Policy, University of Michigan, in the process of revising. This work was supported by the National Natural Science Foundation of China (No. 81572988) and Science \& Technology Department of Sichuan Province Funding Project (Nos. 2016FZ0108, 2018SZ0117).

\section{Disclosure}

The authors report no conflicts of interest in this work.

\section{References}

1. Bray F, Ferlay J, Soerjomataram I, et al. Global cancer statistics 2018: GLOBOCAN estimates of incidence and mortality worldwide for 36 cancers in 185 countries. CA Cancer J Clin. 2018;68(6):394-424. doi:10.3322/caac.v68.6

2. Hurwitz H, Fehrenbacher L, Novotny W, et al. Bevacizumab plus irinotecan, fluorouracil, and leucovorin for metastatic colorectal cancer. N Engl J Med. 2004;350(23):2335-2342. doi:10.1056/ NEJMoa032691

3. Peeters M, Price TJ, Cervantes A, et al. Randomized phase III study of panitumumab with fluorouracil, leucovorin, and irinotecan (FOLFIRI) compared with FOLFIRI alone as second-line treatment in patients with metastatic colorectal cancer. $J$ Clin Oncol. 2010;28 (31):4706-4713. doi:10.1200/JCO.2009.27.6055

4. Van Cutsem E, Kohne CH, Hitre E, et al. Cetuximab and chemotherapy as initial treatment for metastatic colorectal cancer. $N$ Engl J Med. 2009;360(14):1408-1417. doi:10.1056/NEJMoa0805019

5. Bokemeyer C, Bondarenko I, Hartmann JT, et al. Efficacy according to biomarker status of cetuximab plus FOLFOX-4 as first-line treatment for metastatic colorectal cancer: the OPUS study. Ann Oncol. 2011;22 (7):1535-1546. doi:10.1093/annonc/mdq632

6. Bokemeyer C, Bondarenko I, Makhson A, et al. Fluorouracil, leucovorin, and oxaliplatin with and without cetuximab in the first-line treatment of metastatic colorectal cancer. J Clin Oncol. 2009;27 (5):663-671. doi:10.1200/JCO.2008.20.8397
7. Bokemeyer C, Kohne CH, Ciardiello F, et al. FOLFOX4 plus cetuximab treatment and RAS mutations in colorectal cancer. Eur J Cancer. 2015;51(10):1243-1252. doi:10.1016/j.ejca.2015.04.007

8. Tveit KM, Guren T, Glimelius B, et al. Phase III trial of cetuximab with continuous or intermittent fluorouracil, leucovorin, and oxaliplatin (Nordic FLOX) versus FLOX alone in first-line treatment of metastatic colorectal cancer: the NORDIC-VII study. J Clin Oncol. 2012;30(15):1755-1762. doi:10.1200/JCO.2011.38.0915

9. Qin S, Li J, Wang L, et al. Efficacy and tolerability of first-line cetuximab plus leucovorin, fluorouracil, and oxaliplatin (FOLFOX-4) versus FOLFOX-4 in patients with RAS wild-type metastatic colorectal cancer: the open-label, randomized, Phase III TAILOR trial. J Clin Oncol. 2018;36:3031-3039.

10. Venook AP, Niedzwiecki D, Lenz HJ, et al. Effect of first-line chemotherapy combined with cetuximab or bevacizumab on overall survival in patients with KRAS wild-type advanced or metastatic colorectal cancer: a randomized clinical trial. JAMA. 2017;317 (23):2392-2401. doi:10.1001/jama.2017.7105

11. Purmonen T, Martikainen JA, Soini EJ, et al. Economic evaluation of sunitinib malate in second-line treatment of metastatic renal cell carcinoma in Finland. Clin Ther. 2008;30(2):382-392. doi:10.1016/ j.clinthera.2008.02.013

12. Sonnenberg FA, Beck JR. Markov models in medical decision making: a practical guide. Med Decis Making. 1993;13(4):322-338. doi:10.1177/0272989X9301300409

13. Huang J, Liao W, Zhou J, et al. Cost-effectiveness analysis of adjuvant treatment for resected pancreatic cancer in China based on the ESPAC-4 trial. Cancer Manag Res. 2018;10:4065-4072. doi:10.2 147/CMAR

14. Ramsey SD, Andersen MR, Etzioni R, et al. Quality of life in survivors of colorectal carcinoma. Cancer. 2000;88(6):1294-1303. doi:10.1002/(ISSN)1097-0142

15. Riesco-Martinez MC, Berry SR, Ko YJ, et al. Cost-effectiveness analysis of different sequences of the use of epidermal growth factor receptor inhibitors for wild-type KRAS unresectable metastatic colorectal cancer. J Oncol Pract. 2016;12(6):e710-e723. doi:10.1200/JOP.2015.008730

16. Westwood M, van Asselt T, Ramaekers B, et al. KRAS mutation testing of tumours in adults with metastatic colorectal cancer: a systematic review and cost-effectiveness analysis. Health Technol Assess. 2014;18(62):1-132. doi:10.3310/hta18620

17. Murray CJ, Evans DB, Acharya A, et al. Development of WHO guidelines on generalized cost-effectiveness analysis. Health Econ. 2000;9(3):235-251. doi:10.1002/(SICI)1099-1050(200004)9:3<235:: AID-HEC502>3.0.CO;2-O

18. Ocvirk J, Brodowicz T, Wrba F, et al. Cetuximab plus FOLFOX6 or FOLFIRI in metastatic colorectal cancer: CECOG trial. World J Gastroenterol. 2010;16(25):3133-3143. doi:10.3748/wjg.v16.i25.3133

19. Huxley N, Crathorne L, Varley-Campbell J, et al. The clinical effectiveness and cost-effectiveness of cetuximab (review of technology appraisal no. 176) and panitumumab (partial review of technology appraisal no. 240) for previously untreated metastatic colorectal cancer: a systematic review and economic evaluation. Health Technol Assess. 2017;21(38):1-294. doi:10.3310/hta21380

20. Davari M, Ashrafi F, Maracy M, et al. Cost-effectiveness analysis of cetuximab in treatment of metastatic colorectal cancer in Iranian pharmaceutical market. Int J Prev Med. 2015;6:63. doi:10.4103/ 2008-7802.161068

21. Wu B, Yao Y, Zhang K, et al. RAS testing and cetuximab treatment for metastatic colorectal cancer: a cost-effectiveness analysis in a setting with limited health resources. Oncotarget. 2017;8 (41):71164-71172. doi:10.18632/oncotarget. 17029

22. Wen F, Yang Y, Zhang P, et al. Cost-effectiveness of RAS screening before monoclonal antibodies therapy in metastatic colorectal cancer based on FIRE3 study. Cancer Biol Ther. 2015;16(11):1577-1584. doi:10.1080/15384047.2015.1095398 


\section{Publish your work in this journal}

Cancer Management and Research is an international, peer-reviewed open access journal focusing on cancer research and the optimal use of preventative and integrated treatment interventions to achieve improved outcomes, enhanced survival and quality of life for the cancer patient.
The manuscript management system is completely online and includes a very quick and fair peer-review system, which is all easy to use. Visit http://www.dovepress.com/testimonials.php to read real quotes from published authors.

Submit your manuscript here: https://www.dovepress.com/cancer-management-and-research-journal 